VoL. 47 (1993) [259-271]

\title{
ON LOCALLY LIPSCHITZ VECTOR-VALUED INVEX FUNCTIONS
}

\author{
N.D. YEN AND P.H. SACH
}

\begin{abstract}
The four types of invexity for locally Lipschitz vector-valued functions recently introduced by $T$. W. Reiland are studied in more detail. It is shown that the class of restricted $K$-invex in the limit functions is too large to obtain desired optimisation theorems and the other three classes are contained in the class of functions which are invex 0 in the sense of our previous joint paper with B. D. Craven and T. D. Phuong. We also prove that the extended image of a locally Lipschitz vector-valued invex function is pseudoconvex in the sense of $\mathrm{J}$. Borwein at each of its points.
\end{abstract}

\section{INTRODUCTION}

Assume that $X=R^{n}, Y=R^{m}$, and $K \subset Y$ is a closed convex cone. A real-valued differentiable function $f: X \rightarrow R$ is said to be invex at $u \in X$ if for each $x \in X$ there exists $\eta=\eta(x, u)$ such that

$$
f(x)-f(u) \geqslant f^{\prime}(u) \eta
$$

where $f^{\prime}(u)$ is the Frechet derivative of $f$ at $u$. We say that $f$ is invex on $X$ if it is invex at every $u \in X$. Every differentiable convex function defined on $X$ is invex on the whole space. The above generalisation of convexity is due to Hanson [7] who showed that the converse Kuhn-Tucker optimality condition [8] and the duality theorem of Wolfe [16] are still valid for programs with invex functions. According to Craven [4], a differentiable vector-valued function $f: X \rightarrow Y$ is said to be invex (with respect to the given convex cone $K$ ) at $u \in X$ if for every $x \in X$ there exists $\eta$ such that

$$
f(x)-f(u)-f^{\prime}(u) \eta \in K .
$$

It is clear that (1) is a special case of (2) with $Y=R$ and $K=R_{+}$(the nonnegative half-line). Invexity of functions with respect to a cone has proved to be useful for establishing sufficient optimality conditions and duality in vector optimisation. The

Received 16th March, 1992.

We are indebted to Dr. BD Craven for many stimulating discussions on the subject during his visit to Hanoi in September 1991.

Copyright Clearance Centre, Inc. Serial-fee code: 0004-9729/93 \$A2.00+0.00. 
reader is referred to [6] for a recent development of this concept, its application to vector optimisation, and for further references.

Many authors have been interested in weakening the differentiability requirement in (1) and (2). Craven and Glover [5] studied quasidifferentiable invex functions. Reiland [10], Tanaka [14], Tanaka, Fukushima and Ibaraki [15] considered the class of locally Lipschitz real-valued invex functions. In [11] Reiland defined four types of invexity for locally Lipschitz vector-valued functions and obtained some optimisation results for problems with locally Lipschitz data. Recently, Sach and Craven [12, 13] have introduced two classes of invex multifunctions called invex 1 and invex 2 , where the Aubin-Ekeland derivative of multifunctions [1] replaces the Frechet derivative in (1) and (2). That approach unifies many of the known results concerning invexity. A new development of the results on invexity for multifunctions is presented in [6] where invex 1 and invex 2 are proved to be the same and a larger class of invex multifunctions called invex 0 multifunctions, together with its application, is examined.

The aim of this paper is to investigate in more detail the four types of invexity introduced by Reiland in [11]. In Section 2 we apply the concepts of invexity for multifunctions [6] to locally Lipschitz vector-valued functions in order to get a better diagram of relationships between Reiland's four types of invexity. (A part of this diagram was obtained by Reiland [11], Sach and Craven [12]). Namely, we show that the class of functions which are restricted $K$-invex in the limit is too large to have a successful extention of theorems like the converse Kuhn-Tucker property [8], Wolfe's duality [16], and Mond-Weir's duality [9]. Our diagram says that the other three Reiland's classes of locally Lipschitz vector-valued functions are contained in the larger class of locally Lipschitz vector-valued functions which are invex 0 in the sense of [6]. Therefore, a part of Reiland's optimisation results [11] could be improved by using the corresponding results in [6] which were obtained for problems in a more general setting. Section 3 is devoted to a useful geometric property resulting from invexity. According to Borwein [2] a subset $C \subset Y$ is said to be pseudoconvex at $a \in C$ if $C-a \subset \overline{c o} T_{C}^{\prime}(a)$, where $T_{C}^{\prime}(a)$ stands for the Bouligand tangent cone to $C$ at $a$ and symbol $\overline{c o} A$ denotes the closure of the convex hull of $A$. We shall prove that if a locally Lipschitz vector-valued function $f: X \rightarrow Y$ is invex 0 on $X$ with respect to the cone $K \subset Y$ then its extended image, that is, the set $C:=f(X)+K$, is pseudoconvex at each point $a \in C$.

\section{RELATIONSHIP BETWEEN VARIOUS TYPES OF INVEXITY}

Let $X, Y$ and $K$ be given as in the Introduction. Consider a vector-valued function $f: X \rightarrow Y$. We say that $f$ is locally Lipschitz at a point $u \in X$ if and only if there exists a number $l>0$ such that $\left\|f(x)-f\left(x^{\prime}\right)\right\| \leqslant l\left\|x-x^{\prime}\right\|$ for all $x, x^{\prime}$ in a neighbourhood of $u$. Function $f$ is said to be locally Lipschitz on a subset $X_{1} \subset X$ if it 
is locally Lipschitz at each point of $X_{1}$. Denote by $f_{i}(i=1,2, \ldots, m)$ the components of $f$, that is $f=\left(f_{1}, \ldots, f_{m}\right)$ and each $f_{i}$ is a real-valued function defined on $X$. Let us recall the concepts of generalised Jacobian and generalised gradient of a locally Lipschitz function.

DEFINITION 1: [3] The generalised Jacobian of $f$ at $u$, denoted by $J f(u)$, is the convex hull of all limits of the form:

$$
A=\lim _{x_{i} \rightarrow x_{i} ; x_{i} \in D_{f}} f^{\prime}\left(x_{i}\right)
$$

where $D_{f}$ is the set of all $x$ where $f^{\prime}(x)$ exists.

Recall [3] that $J f(u)$ is a compact convex subset of the space $L(X, Y)$ of linear operators from $X$ to $Y$.

Given a real-valued function $\varphi: X \rightarrow R$ which is assumed to be locally Lipschitz at a point $u \in X$ we define, as in [3], the generalised directional derivative $\varphi^{\circ}(u ; v)$ of $\varphi$ at $u$ in the direction $v \in X$ by setting

$$
\varphi^{\circ}(u ; v)=\limsup _{t \rightarrow 0+; x \rightarrow u} t^{-1}[\varphi(x+t v)-\varphi(x)]
$$

The generalised gradient [3] of $\varphi$ at $u$ is the following set

$$
\partial \varphi(u)=\left\{\eta \in X:\langle\eta, v\rangle \leqslant \varphi^{\circ}(u ; v) \text { for every } v \in X\right\}
$$

where $\langle.,$.$\rangle denotes the scalar product in X$. It has been proved [3] that $\partial \varphi(u)=J \varphi(u)$.

DEFINITON 2: $[3,11]$ The generalised gradient of $f$ at $u$ is the set

$$
\partial f(u)=\partial f_{1}(u) \times \ldots \times \partial f_{m}(u)
$$

where $\partial f_{i}($.$) is the generalised gradient of f_{i}$ as defined above.

Every element

$$
A=\left(\nu_{1}, \ldots, \nu_{m}\right) \in \partial f(u)
$$

is interpreted as an operator from $L(X, Y)$ by setting

$$
A x=\left(\left\langle\nu_{1}, x\right\rangle, \ldots,\left\langle\nu_{m}, x\right\rangle\right) \in Y=R^{m}
$$

for all $x \in X$. (Here and in the sequel we make no distinction between a row-vector and the corresponding column-vector.) It is known [3] that $\partial f(u)$ is also a convex compact subset of $L(X, Y)$. Moreover, $J f(u) \subset \partial f(u)$, and there exist examples for which $J f(u) \neq \partial f(u)$. For each vector $w \in X$ we define a function: $f_{w}()=.\langle w, f()$.$\rangle .$ 
We now turn to the definition of Reiland's four types of invexity.

DEFINITION 3: [11]

A. $f$ is $K$-invex at $u$ if for every $x \in X$ there exists $\eta \in X$ such that $\forall A \in \partial f(u) \quad f(x)-f(u)-A \eta \in K$.

B. $f$ is $K$-invex in the limit at $u$ if for every $x \in X$ there exists $\eta \in X$ such that

where

$$
f(x)-f(u)-f^{\circ}(u ; \eta) \in K,
$$

where $f^{o}(u ; \eta)=\left(f_{1}^{o}(u, \eta), \ldots, f_{m}^{o}(u, \eta)\right)$.

C. $f$ is restricted. $K$-invex at $u$ if for every $x \in X$ there exists $\eta \in X$ such that

$$
\forall A \in J f(u) \quad f(x)-f(u)-A \eta \in K .
$$

D. $f$ is restricted $K$-invex in the limit at $u$ if for every $x \in X$ there exists $\eta \in X$ such that

$$
\forall w \in K^{-} \quad\langle w, f(x)-f(u)\rangle \leqslant f_{w}^{o}(u ; \eta),
$$

$$
K^{-}=\{w \in Y:\langle w, y\rangle \leqslant 0, \text { for all } y \in K\} \text {. }
$$

As in [6] we set $\hat{f}()=.f()+$.$K and denote by D \hat{f}_{(u, f(u))}($.$) the Aubin-Ekeland$ derivative [1] of the set-valued map (multifunction) $\hat{f}$ at the point $(u, f(u)) \in g r \widehat{f}:=$ $\{(x, y) \in X \times Y: y \in \widehat{f}(x)\}$. This means that

$$
D \widehat{f}_{(u, f(u))}(x)=\left\{y:(x, y) \in T_{g r \hat{f}}(u, f(u))\right\},
$$

where $T_{g r \hat{f}}(u, f(u))$ is the Clarke tangent cone to $g r \hat{f}$ at $(u, f(u)$ ) (see Definition 5 in Section 3).

We can define invexity for locally Lipschitz vector-valued functions in two other ways, as follows.

DEFINITION 4: [6]

E. $f$ is invex 1 at $u$ if for every $x \in X$ there exists $\eta \in X$ satisfying

$$
f(x)-f(u) \in D \widehat{f}_{(u, f(u))}(\eta) .
$$

F. $f$ is invex 0 at $u$ if for each $x \in X$ we have

$$
f(x)-f(u) \in \overline{D \widehat{f}_{(u, f(u))}(X)},
$$


where $D \hat{f}_{(u, f(u))}(X)=\bigcup\left\{D \hat{f}_{(u, f(u))}(x): x \in X\right\}$ and the bar denotes the closure hull.

Lemma 1. The function $f$ is invex 1 at $u$ if and only if : $\forall x \in X \exists \eta \in X$ satisfying

$$
\langle w, f(x)-f(u)\rangle \geqslant f_{w}^{o}(u ; \eta), \quad \forall w \in K^{+}:=-K^{-}
$$

PROOF: Indeed, applying [12, Lemma 11] yields

$$
D \widehat{f}_{(u, f(u))}(\eta)=\left\{y \in Y:\langle w, y\rangle \geqslant f_{w}^{o}(u ; \eta) \text { for all } w \in K^{+}\right\}
$$

Therefore, (9) is equivalent to (7), and the conclusion follows.

REMARK 1. The formula (10) is useful for computing the image set $D \widehat{f}_{(u, f(u))}(X)$, and, therefore, to verify the invex 0 property described in (8).

REMARK 2. [6] If $f$ is assumed to be a map of $C^{1}$-class (which consists of continuously differentiable functions), then (7) and (8) become, respectively,

and

$$
\begin{aligned}
& f(x)-f(u) \in f^{\prime}(u) \eta+K \\
& f(x)-f(u) \in \overline{f^{\prime}(u)(X)+K} .
\end{aligned}
$$

These formulas show that invexity in the sense of Definition 4 is an extension of that one proposed by Craven [4], which has been recalled in (2).

Our task is to describe relationships between the various types of invexity for locally Lipschitz vector-valued functions given in Definition 3 and Definition 4. This work has been done partially by Reiland [11] and Sach and Craven [12].

THEOREM 1. The relationships between the definitions of invexity $(A)-(F)$ are given by the following diagram

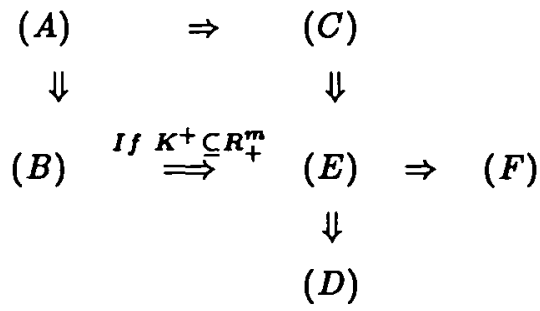

Diagram 1 
If $K^{+} \subseteq R_{+}^{m}$ then $(A) \Leftrightarrow(B)$ and Diagram 1 is simplified as follows :

$$
\begin{gathered}
(A) \Rightarrow(C) \Rightarrow(E) \Rightarrow(F) \\
\Downarrow
\end{gathered}
$$

\section{Diagram 2}

Proof: $(A) \Rightarrow(C)$ : Since $J f(u) \subset \partial f(u)$, this implication is trivial.

$(C) \Rightarrow(E)$ : Assume that $f$ is restricted $K$-invex at $u$. Given any $x \in X$ we choose $\eta \in X$ satisfying (5). We claim that this $\eta$ satisfies (9). Indeed, for every $w \in K^{+}$applying (5) one gets

$$
\langle w, f(x)-f(u)\rangle \geqslant \beta:=\sup \{(w, A \eta\rangle: A \in J f(u)\} .
$$

Fixing an arbitrary $\varepsilon>0$, by virtue of [3, Proposition 2.6.2] there exists a convex neighbourhood $U$ of $u$ such that

$$
\forall u^{\prime} \in U, \quad J f\left(u^{\prime}\right) \subset J f(u)+\varepsilon B_{L(X, Y)},
$$

where $B_{L(X, Y)}$ is the unit ball in $L(X, Y)$. Using the mean-value theorem in [3, Proposition 2.6.5], for all $u^{\prime}, u^{\prime \prime} \in U$, we obtain from (12) that

$$
\begin{aligned}
f\left(u^{\prime \prime}\right)-f\left(u^{\prime}\right) & \subset \operatorname{coJ} f\left(\left[u^{\prime}, u^{\prime \prime}\right]\right)\left(u^{\prime \prime}-u^{\prime}\right) \\
& \subset\left[J f(u)+\varepsilon B_{L(X, Y)}\right]\left(u^{\prime \prime}-u^{\prime}\right) .
\end{aligned}
$$

(The symbol $c o E$ denotes the convex hull of $E$ ). Therefore,

$$
\begin{aligned}
f_{w}^{o}(u ; \eta) & =\limsup _{t \rightarrow 0+; u^{\prime} \rightarrow u} t^{-1}\left[\left\langle w, f\left(u^{\prime}+t \eta\right)-f\left(u^{\prime}\right)\right\rangle\right] \\
& \leqslant \sup \left\{\langle w, A \eta+\varepsilon M \eta\rangle: A \in J f(u), M \in B_{L(X, Y)}\right\} \\
& =\beta+\varepsilon\|w\|\|\eta\| .
\end{aligned}
$$

Since $\varepsilon$ is arbitrarily chosen, we have $f_{w}^{o}(u ; \eta) \leqslant \beta$. Now it is clear that $(9)$ follows from (11). We have thus proved the implication.

$(E) \Rightarrow(D)$ : Multiplying both sides of $(6)$ by -1 , we have the following inequality

$$
\langle-w, f(x)-f(u)\rangle \geqslant \liminf _{t \rightarrow 0+; u^{\prime} \rightarrow u} t^{-1}\left[\left\langle-w, f\left(u^{\prime}+t \eta\right)-f\left(u^{\prime}\right)\right\rangle\right] .
$$

Hence, $f$ is restricted K-invex in the limit at $u$ if and only if for every $x \in X$ there is $\eta \in X$ such that

$$
\forall w \in K^{+} \quad\langle w, f(x)-f(u)\rangle \geqslant \liminf _{t \rightarrow 0+; u^{\prime} \rightarrow u^{-1}} t^{-1}\left[\left\langle w, f\left(u^{\prime}+t \eta\right)-f\left(u^{\prime}\right)\right\rangle\right] .
$$


By Lemma $1, f$ is invex 1 at $u$ if and only if for every $x \in X$ there exists $\eta \in X$ satisfying

$$
\forall w \in K^{+} \quad\langle w, f(x)-f(u)\rangle \geqslant \limsup _{t \rightarrow 0+; u^{\prime} \rightarrow u} t^{-1}\left[\left(w, f\left(u^{\prime}+t \eta\right)-f\left(u^{\prime}\right)\right\rangle\right]
$$

It is a simple matter to obtain the desired implication from (13) and (14).

$(A) \Rightarrow(B)$ : Let $f$ be $K$-invex at $u$. Given $x \in X$ we pick $\eta \in X$ satisíying (3). If for each index $i \in\{1, \cdots, m\}$ we choose $\bar{\nu}_{i} \in \partial f_{i}(u)$ such that $\left\langle\bar{\nu}_{i}, \eta\right\rangle=\sup \left\{\left\langle\nu_{i}, \eta\right\rangle\right.$ : $\left.\nu_{i} \in \partial f_{i}(u)\right\}=f_{i}^{\circ}(u ; \eta)$, then $\bar{A}:=\left(\bar{\nu}_{1}, \cdots, \bar{\nu}_{m}\right) \in \partial f(u)$. Substituting $\bar{A}$ in (3) yields (4) which means that $f$ is $K$-invex in the limit at $u$.

$(B) \Rightarrow(E)$ (Under the condition $K^{+} \subset R_{+}^{m}$ ): Assume that $f$ is $K$-invex in the limit at $u$. Given $x \in X$ choose $\eta \in X$ such that (4) holds. Then for any $w=\left(w_{1}, \cdots, w_{m}\right) \in K^{+}$we have

$$
\begin{aligned}
\langle w, f(x)-f(u)\rangle & \geqslant \sum_{i=1}^{m} w_{i} f_{i}^{o}(u ; \eta) \\
& =\sum_{i=1}^{m} w_{i} \sup \left\{\left\langle\nu_{i}, \eta\right\rangle: \nu_{i} \in \partial f_{i}(u)\right\}
\end{aligned}
$$

On the other hand, since $w \in K^{+} \subset R_{+}^{m}$ we have

$$
\begin{aligned}
f_{w}^{o}(u ; \eta) & =\sup \left\{\langle\nu, \eta\rangle: \nu \in \partial\left(\sum_{i=1}^{m} w_{i} f_{i}\right)(u)\right\} \\
& \leqslant \sup \left\{\langle\nu, \eta\rangle: \nu \in \sum_{i=1}^{m} w_{i} \partial f_{i}(u)\right\} \\
& =\sum_{i=1}^{m} w_{i} \sup \left\{\left\langle\nu_{i}, \eta\right\rangle: \nu_{i} \in \partial f_{i}(u)\right\}
\end{aligned}
$$

Combining this and (15) gives

$$
\langle w, f(x)-f(u)\rangle \geqslant f_{w}^{o}(u ; \eta)
$$

which proves that $f$ is invex 1 at $u$.

$(E) \Rightarrow(F)$ : This implication is obvious from Definition 4.

Now, suppose that $K^{+} \subset R_{+}^{m}$. If we can show that $(B) \Rightarrow(A)$, then $(A) \Leftrightarrow(B)$ and Diagram 1 reduces to Diagram 2. To this end, we suppose that $f$ is $K$-invex in the limit at $u$, that is : $\forall x \in X \exists \eta \in X$ such that (4) holds. For each $w=\left(w_{1}, \cdots, w_{m}\right) \in$ 
$K^{+} \subset R_{m}^{+}$, from the (4) we can deduce that

$$
\begin{aligned}
& \left\langle w, f(x)-f(u)-f^{\circ}(u ; \eta)\right\rangle \\
& =\langle w, f(x)-f(u)\rangle-\sum_{i=1}^{m} w_{i} \sup \left[\left\langle\nu_{i}, \eta\right\rangle: \nu_{i} \in \partial f_{i}(u)\right] \\
& =\inf \left\{\langle w, f(x)-f(u)\rangle-\sum_{i=1}^{m} w_{i}\left(\nu_{i}, \eta\right\rangle: \nu_{i} \in \partial f_{i}(u), i=1,2, \ldots, m\right\} \\
& =\inf [\langle w, f(x)-f(u)-A \eta\rangle: A \in \partial f(u)] \geqslant 0 .
\end{aligned}
$$

This implies that $f$ is $K$-invex at $u$. The proof is complete.

REMARK 3. If $Y=R, K=R_{+}$and $f$ is locally Lipschitz real-valued function, then all the notions (A), (B), (C), (E), (F) coincide. Indeed, given any $u \in X$, from the definitions, Lemma 1 , and the equality $f^{\circ}(u ; \eta)=\max \{\langle\nu, \eta\rangle: \nu \in \partial f(u)\}$, it follows that that the properties described by (A), (B), (C), and (E), are the same. It remains to add [6] that a locally Lipschitz real-valued function $f$ is invex 1 at $u$ if and only if it is invex 0 at $u$.

We are going to comment on the above diagrams by some examples. The following example shows that the restricted $K$-invex in the limit property does not imply invex 0 and invex 1 .

EXAmPle 1. $[(D) \nRightarrow(F) ;(D) \nRightarrow(E)]$ Let $X=R^{2}, Y=R, K=R_{+}$and $f: X \rightarrow Y$ be given by $f(x)=\max \left[\min \left(x_{1},-x_{2}\right), x_{2}-x_{1}\right]$ for all $x=\left(x_{1}, x_{2}\right) \in X$ (see Clarke [5, Example 2.5.2]). To simplify the formula for $f(x)$ we divide the plane $X=R^{2}$ into four parts :

$$
\begin{aligned}
& D_{1}=\left\{x: x_{2} \leqslant-x_{1}, x_{2} \geqslant 2 x_{1}\right\}, \\
& D_{2}=\left\{x: x_{2} \leqslant-x_{1}, x_{2} \leqslant 2 x_{1}\right\}, \\
& D_{3}=\left\{x: x_{2} \geqslant-x_{1}, x_{2} \geqslant 2^{-1} x_{1}\right\}, \\
& D_{4}=\left\{x: x_{2} \geqslant-x_{1}, x_{2} \leqslant 2^{-1} x_{1}\right\} ;
\end{aligned}
$$

and then note that: $f(x)=x_{2}-x_{1}$ for $x \in D_{1}, f(x)=x_{1}$ for $x \in D_{2}, f(x)=x_{2}-x_{1}$ for $x \in D_{3}, f(x)=-x_{2}$ for $x \in D_{4}$. Since $\partial f(\bar{x})=c o\left\{\lim _{\xi_{i} \rightarrow \bar{x} ; \xi_{i} \in D_{f}} f^{\prime}\left(\xi_{i}\right)\right\}$, it is easy to compute the generalised gradient of $f$ at each point $x \in X$. Especially,

$$
\partial f(0)=\operatorname{co}\{(1,0),(0,-1),(-1,1)\} .
$$

Therefore, $0 \in \operatorname{int} \partial f(0)$ and there exists $\rho>0$ such that $\rho \bar{B} \subset \partial f(0)$, where $\bar{B}$ stands for the unit ball in $X$. We infer that $f$ is restricted $K$-invex in the limit at $u=0$. Indeed, condition (6) can be rewritten as : $\forall x \in X \quad \exists \eta \in X$ such that

$$
\langle w, f(x)-f(0)\rangle \geqslant-f_{w}^{o}(0 ;-\eta), \quad \forall w \in K^{+}=R_{+} .
$$


This is equivalent to saying that: $\forall x \in X \exists \eta \in X$ such that:

$$
f(x) \geqslant-f^{\circ}(0 ;-\eta)
$$

Given any $x \in X$ we choose $\eta \in X$ such that

$$
f(x) \geqslant-\rho\|\eta\| \text {. }
$$

Taking account of the fact that $\rho \bar{B} \subset \partial f(0)$, we have

$$
\begin{aligned}
-f^{o}(0 ;-\eta) & =-\max _{\nu \in \theta f(0)}\langle\nu,-\eta\rangle \\
& \leqslant-\max _{\nu \in \rho \bar{B}}\langle\nu,-\eta\rangle \\
& =-\rho\|\eta\| .
\end{aligned}
$$

Combining this with (17) gives (16) which proves restricted $K$-invexity in the limit of $f$ at $u=0$. Furthermore, we claim that $f$ is not invex 0 at $u=0$. Indeed, since $f$ is a locally Lipschitz real-valued function, according to a result in [6, Proposition 2.4] $f$ is invex 0 at $u=0$ if and only if it is invex 1 at this point. So, to prove the claim it suffices to find a point $x \in X$ such that for some $w \in K^{+}=R_{+}$there exists no $\eta \in X$ satisfying

$$
\langle w, f(x)-f(0)\rangle \geqslant f_{w}^{o}(0 ; \eta) .
$$

Using the formula of $f$ we can find $x \in X$ such that $f(x)<0$ (for example, $x$ could be any point of the form $x=(-\tau,-2 \tau), \tau>0$, which lies on the common boundary of $D_{1}$ and $D_{2}$ ). On the other hand, the inclusion $0 \in \partial f(0)$ implies that if we take $w=1$ then $f_{w}^{o}(0 ; \eta) \geqslant 0$ for all $\eta \in X$. Therefore, $(18)$ is not satisfied with any vector $\eta \in X$. By this example we have shown that $(D) \nRightarrow(F)$, and, hence, $(D) \nRightarrow(E)$.

A careful analysis of the above counterexample allows us to construct another simplier one serving the same purpose.

Example 2. Let $X=Y=R, K=R_{+}$, and $f: X \rightarrow Y$ be defined by setting $f(x)=x^{2}-|x|, \forall x \in X$. It is clear that $f$ is an even function having three zero points $0,1,-1$. For $u=0$ we have $\partial f(u)=[-1,1]$. Obviously, there exists $x \in X$ such that $f(x)<0$. Arguing similarly as in Example 1 we conclude that $f$ is restricted $K$-invex in the limit at $u$ but $f$ is not invex 0 at this point. (It is worth noting that our function is restricted $K$-invex in the limit at every point of $X$.)

EXAmple 3. With the notation in Example 2, consider the following minimisation problem $(P)$ :

$$
\begin{array}{ll}
\text { Minimise } & f(x) \\
\text { subject to } & x \in X .
\end{array}
$$


Since we have $0 \in \partial f(0)$, then 0 is a $K u h n$-Tucker stationary point of $(P)$. As it has been noted, $f$ is restricted $K$-invex in the limit at 0 . However, 0 is not a global minimum point of $(P)$. This example shows that minimisation problems with data from the class of restricted $K$-invex in the limit functions may not satisfy the the converse Kuhn-Tucker property which says that : Every Kuhn-Tucker stationary point of the problem is a global minimum. Let us observe [6] that the converse Kuhn-Tucker property holds for minimisation problems whose objective and constraint functions are invex 0 in the sense of Definition 4. The Wolfe dual of (P) (see [10] and [11]) is the following problem (D):

$$
\begin{array}{ll}
\text { Maximise } & f(x) \\
\text { subject to } & x \in X, 0 \in \partial f(x) .
\end{array}
$$

It is quite easy to show that the weak duality relation does not hold between (P) and (D).

EXAMPLE 4. $[(B) \nRightarrow(E)$, for $K$ being in general position]: Let $X=R, Y=$ $R^{2}, f(x)=\left(|x|, 2^{-1} x\right)$ for all $x \in X$. Let $K=\left\{y=\left(y_{1}, y_{2}\right) \in Y: y_{2} \geqslant y_{1} \geqslant 0\right\}$. We have $K^{+}=\{w=(\alpha, \beta): \beta \geqslant-\alpha, \beta \geqslant 0\}$. Observe that $f$ is $K$-invex in the limit at $u=0$. Indeed, condition (4) is now equivalent to the following $: \forall x \in X \exists \eta \in X$ such that

$$
\left(|x|, 2^{-1} x\right)-\left(|\eta|, 2^{-1} \eta\right) \in K
$$

In turn this property can be rewritten as : $\forall x \in X \exists \eta \in X$ such that

$$
2^{-1} x-2^{-1} \eta \geqslant|x|-|\eta| \geqslant 0 \text {. }
$$

It is obvious that the last condition is satisfied with $\eta=x$. Now we have only to show that $f$ is not invex 1 at $u$. Suppose the contrary. Then for $x=1$ there must exist $\eta \in X$ satisfying

$$
\langle w, f(1)-f(0)\rangle \geqslant f_{w}^{o}(0 ; \eta), \quad \forall w \in K^{+} .
$$

Consequently, for $\bar{w}=(-1,1) \in K^{+}$we have $\langle\bar{w}, f(1)\rangle \geqslant f_{\bar{w}}^{o}(0 ; \eta)$. Since $f_{\bar{w}}(x)=$ $-|x|+2^{-1} x$, then $f_{w}^{o}(0 ; \eta)=|\eta|+2^{-1} \eta$. Therefore, $-|1|+2^{-1} \geqslant|\eta|+2^{-1} \eta$. The left-hand side of this inequality is less than 0 , while the right-hand side is nonnegative, a contradiction.

EXAMPLE 5. [6] $[(F) \nRightarrow(E)]$. Let $X=R, Y=R^{3}, f(x)=\left(x, x^{3}, 0\right), u=0, K=\{y=$ $\left.(\alpha, \beta, \gamma) \in R^{3}:\left(\gamma-2^{-1} \alpha\right)^{2}+\beta^{2} \leqslant 4^{-1} \alpha^{2}\right\}$. We have $f^{\prime}(u)(X)=\{y=(\alpha, 0,0):$ $\alpha \in R\}$. An easy computation shows that

$$
\begin{aligned}
& f^{\prime}(u)(X)+K=\{y=(\alpha, \beta, \gamma): \gamma>0\} \cup\{y=(\alpha, \beta, \gamma): \gamma=\beta=0\}, \\
& \overline{f^{\prime}(u)(X)+K}=\{y=(\alpha, \beta, \gamma): \gamma \geqslant 0\} .
\end{aligned}
$$


Then we obtain that $f^{\prime}(u)(X)+K$ does not contain the set $f(X)-f(u)$, while $\overline{f^{\prime}(u)(X)+K}$ contains it. By Remark 2 one can see that $f$ is invex 1 but not invex 0 at $u$.

Remark 4. Note that the converse Kuhn-Tucker, the Wolfe duality and the MondWeir duality have been obtained [6] for optimisation problems with invex 0 functions. Then, using Diagram 1 we can deduce a part of Reiland's results in [11] from the results in [6]. Since giving more details would require a lot of space we restrict ourselves to this observation. Another meaning of Diagram 1 is that (A), (B), (C), and (E) could be considered as sufficient conditions for the weaker invex property (F) (which we name by invex 0 ). Of course, computation based on formulas (3)-(5) and information about the sets $J f(u)$ and $\partial f(u)$, in many cases, is much more easiser than that using generalised directional derivatives and formulas (8),(10).

REMARK 5. If the condition $K \subset R_{+}^{m}$ is violated, then we do not have that $(A) \Leftrightarrow(B)$. EXAMPLE 6. Let $X=Y=R, f(x)=|x|, K=\{x: x \leqslant 0\}$ then $f$ is $K$-invex in the limit at 0 , but not $K$-invex at 0 .

Concerning Theorem 1 we have the following open question :

Question. Is it true that the implications $(A) \Rightarrow(C)$ and $(C) \Rightarrow(E)$ are not reversable in the general case?

\section{ConVEXITY OF THE EXTENDED IMAGE OF INVEX FunCtions}

In this section, adopting all notations of Section 2 we discuss a convexity property of the set $f(X)+K$ when $f$ is assumed to be invex 0 on $\mathrm{X}$. Note that invex 0 is defined for an arbitrary function, but in this section we restrict ourselves to continuous function $f$. Also, Theorem 2 below can be established for the set-valued case.

We first recall the definitions of Clarke's and Bouligand's tangent cones.

Definition 5: $[1,3]$ Clarke's tangent cone $T_{C}(a)$ to a subset $C$ in a Banach space $Z$ at a point $u \in C$ is the set of all vectors $v \in Z$ such that for each sequence $\left\{a_{i}\right\} \subset C, a_{i} \rightarrow a$, and each sequence $\left\{t_{i}\right\} \subset R_{+}, t_{i} \rightarrow 0$, there exists a sequence $\left\{v_{i}\right\} \subset Z, v_{i} \rightarrow v$, such that $a_{i}+t_{i} v_{i} \in C$ for each integer $i$.

Definition 6: $[1,3]$ Bouligand's tangent cone $T_{C}^{\prime}(a)$ to a subset $C \subset Z$ at a point $a \in C$ is the set of all vectors $v \in Z$ such that there exist a sequence of vectors $\left\{v_{i}\right\} \subset Z, v_{i} \rightarrow v$ and a sequence $\left\{t_{i}\right\} \subset R_{+}, t_{i} \rightarrow 0$ such that $a+t_{i} v_{i} \in C$ for each integer $i$.

Obviously, $T_{C}(a) \subset T_{C}^{\prime}(a)$. According to Borwein [2], a subset $C \subset Z$ is said to be pseudoconvex at a point $a \in C$ if and only if $C-a \subset \overline{c o} T_{C}^{\prime}(a)$. It is clear that every convex set is pseudoconvex at each of its points. The converse statement is false. 
EXAMPLE 7. Let $Y=R^{2}$ and $C=\left\{y=(\alpha, \beta) \in Y: \alpha^{2}+\beta^{2}>1\right\}$. It can be seen that the nonconvex set $C$ is pseudoconvex at each $a \in C$.

LEMMA 2. [2] $A$ closed subset in a reflexive Banach space is convex if and only if it is pseudoconvex at all its points.

Lemma 3. [13, Lemma 5.1] Assume that $f: X \rightarrow Y$ is a continuous vectorvalued function and $K \subset Y$ is a closed convex cone. Then, for every $u \in X$ and $q \in K$ we have

$$
T_{g r \hat{f}}(u, f(u))+\{0\} \times \overline{\operatorname{cone}[K-q]} \subset T_{g r \hat{f}}(u, f(u)+q)
$$

and

$$
D \widehat{f}_{(u, f(u))}(x)+\overline{\text { cone }[K-q]} \subset D \widehat{f}_{(u, f(u)+q)}(x), \forall x \in X
$$

where $\widehat{f}():.=f()+$.$K .$

Note that the properties (19) and (20) are equivalent by definitions, and the inclusion (20) was established in [13].

THEOREM 2. Assume that $f: X \rightarrow Y$ is a continuous vector-valued function and that $f$ is invex 0 (with respect to the cone $K$ ) at every $u \in X$. Then the extended image $C:=f(X)+K$ of $f$ is pseudoconvex at each point $a \in C$. In addition, if $C$ is closed then $C$ is convex.

Proof: Since $f$ is invex 0 on $X$, then

$$
\forall u \in X \quad f(X)-f(u) \subset \overline{D \widehat{f}_{(u, f(u))}(X)}
$$

By [12, Lemma 2] we have from (21) that

$$
f(X)+K-f(u) \subset \overline{D \widehat{f}_{(u, f(u))}(X)}
$$

By virtúe of (20), for every $q \in K, \overline{D \widehat{f}_{(u, f(u))}(X)}-q \subset \overline{D f_{(u, f(u)+q)}(X)}$. Consequently, (20) and (22) imply that

$$
f(X)+K-(f(u)+q) \subset \overline{D \widehat{f}_{(u, f(u))}(X)}-q \subset \overline{D \widehat{f}_{(u, f(u)+q)}(X)} .
$$

We claim that

$$
\overline{D \widehat{f}_{(u, f(u)+q)}(X)} \subset T_{C}^{\prime}(f(u)+q) .
$$

Indeed, since the Bouligand tangent cone is closed, it suffices to show that

$$
D \hat{f}_{(u, f(u)+q)}(X) \subset T_{C}^{\prime}(f(u)+q) \text {. }
$$


Let $v$ belong to the left side of (25). Then there is $\eta \in X$ such that $(\eta, v) \in$ $T_{g r \hat{f}}(u, f(u)+q) \subset T_{g r \hat{f}}^{\prime}(u, f(u)+q)$. Hence, there exist a sequence $\left\{t_{i}\right\} \subset R_{+}, t_{i} \rightarrow 0$, and a sequence $\left\{\left(\eta_{i}, v_{i}\right)\right\} \subset X \times Y,\left(\eta_{i}, v_{i}\right) \rightarrow(\eta, v)$, such that

$$
(u, f(u)+q)+t_{i}\left(\eta_{i}, v_{i}\right) \in g r \widehat{f} .
$$

In other words, $f(u)+q+t_{i} v_{i} \in \widehat{f}\left(u+t_{i} \eta_{i}\right)$ for every integer $i$. Therefore, $f(u)+q+$ $t_{i} v_{i} \in C$ for every integer $i$. This shows that $v$ is an element of the right side of (25), as desired. Combining (23) and (24) implies that $C-a \subset T_{C}^{\prime}(a)$ for each $a \in C$. This implies that $C$ is pseudoconvex at each $a \in C$. If, in addition, $C$ is closed, then $C$ is convex by Lemma 2 .

The following example shows that both $C$ and $\bar{C}$ may be nonconvex while the assumptions of Theorem 2 are valid.

EXAMPLE 8. Let $X=R^{2}, Y=R^{2}, K=\{y=(\alpha, \beta): \alpha=0, \beta \geqslant 0\}$, and $f(x)=$ $\left(x_{1},-x_{1}^{2}+e^{x_{2}}\right)$ for every $x=\left(x_{1}, x_{2}\right) \in X$. Since $f^{\prime}(u)$ is surjective for every $u \in X$, then using Remark 2 we obtain that $f$ is invex 0 at every $u \in X$. Thus the assumptions of Theorem 2 are satisfied. It is clear that $C=\left\{y=(\alpha, \beta) \in Y: \beta>-\alpha^{2}\right\}$ and $\bar{C}=\left\{y=(\alpha, \beta): \beta \geqslant-\alpha^{2}\right\}$ are nonconvex subsets of $Y$.

\section{REFERENCES}

[1] J.P. Aubin and I. Ekeland, Applied nonlinear analysis (Wiley, New York, 1984).

[2] J. Borwein, 'Weak tangent cones and optimization in Banach space', SIAM J. Control Optim. 16 (1978), 512-522.

[3] F.H. Clarke, Optimization and nonsmooth analysis (Wiley, New York, 1983).

[4] B.D. Craven, 'Invex functions and constrained local minima', Bull. Austral. Math. Soc. 24 (1981), 357-366.

[5] B.D. Craven and B.M. Glover, 'Invex functions and duality', J. Austral. Math. Soc. Ser. A 39 (1985), 1-20.

[6] B.D. Craven, P.H. Sach, N.D. Yen and T.D. Phuong, 'A new class of invex multifunctions', Papers presented at the Summer School in Erice (Sicily), June 19-30, 1991; Depart. of Math., Melbourne University, Preprint no.21 (1991), Nonsmooth Optimization : Methods and Applications (to appear).

[7] M.A. Hanson, 'On sufficiency of the Kuhn-Tucker conditions', J. Math. Anal. Appl. 80 (1981), 545-550.

[8] O.L. Mangasarian, Nonlinear programming (McGraw-Hill, New York, 1969).

[9] B. Mond and T. Weir, 'Generalized concavity and duality', in Generalized concavity in optimization and economics, (S. Schaible and W. T. Ziemba, Editors) (Academic Press, New York, 1981), pp. 263-279.

[10] T.W. Reiland, 'Nonsmooth invexity', Bull. Austral. Math. Soc. 42 (1990), 437-446. 
[11] T.W. Reiland, 'Generalized invexity for nonsmooth vector-valued mappings', Numer. Funct. Anal. Optim. 10 (1989), 1191-1202.

[12] P.H. Sach and B.D. Craven, 'Invexity in multifunction optimization', Numer. Funct. Anal. Optim. 12 (1991), 383-394.

[13] P.H. Sach and B.D. Craven, 'Invex multifunctions and duality', Numer. Funct. Anal. Optim. 12 (1991), 575-591.

[14] Y. Tanaka, 'Note on generalized convex functions', J. Optim. Theor. Appl. 66 (1990), 345-349.

[15] Y. Tanaka, M. Fukushima and T. Ibaraki, 'On generalized pseudoconvex functions', J. Math. Anal. Appl. 144 (1989), 342-355.

[16] P. A. Wolfe, 'A duality theorem for nonlinear programming', Quart. Appl. Math. 19 (1961), 239-244.

Institute of Mathematics

PO Box 631, Bo Ho

10000 Hanoi

Vietnam 